\title{
DT
}

650

.$M 64 C 47$

1896

AFA

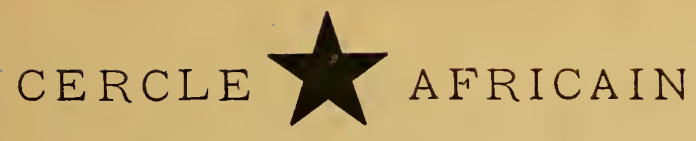

DE BRUXELLES

- 00250

\section{LE PAYS DES MANGBETTUS}

CAUSERIE

Faite aux membres du Cercle, le 28 Novembre 1896

PAR LE

Gapitaine Commandant CHRISTIAENS

ADJOINT D'ÉTAT-MAJOR

BRUXELLES

IMPRIMERIE VAN CAMPENHOUT FRĖRES \& SEUUR

I3, RUE DE LA COLLINE, I3.

1896 

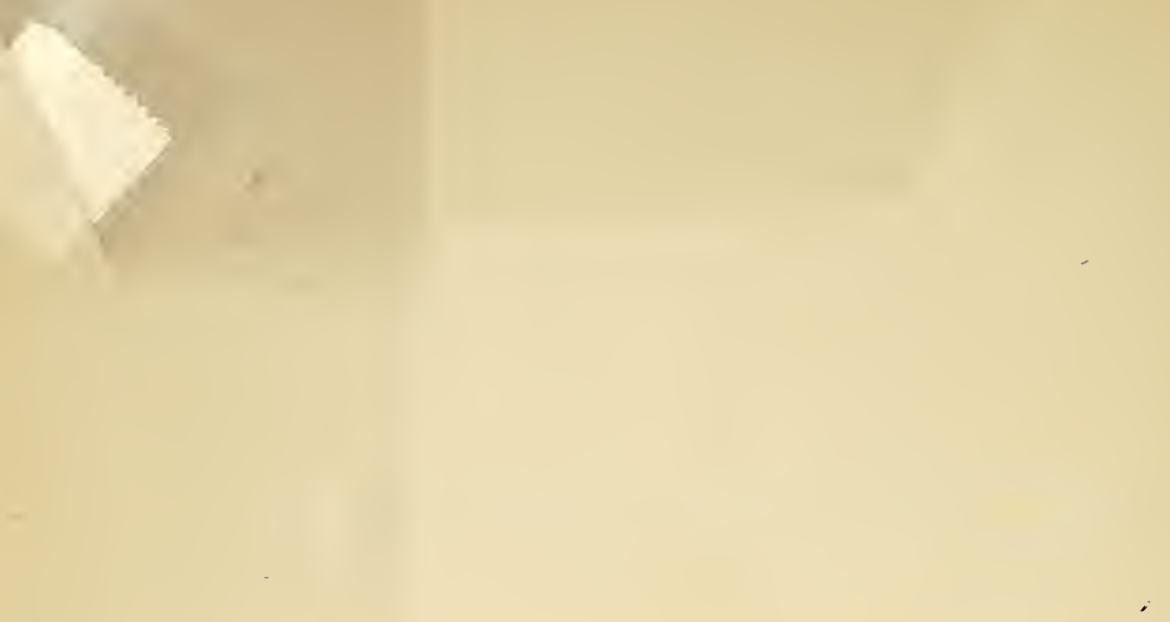
LE PAYS DES MANGBETTUS 



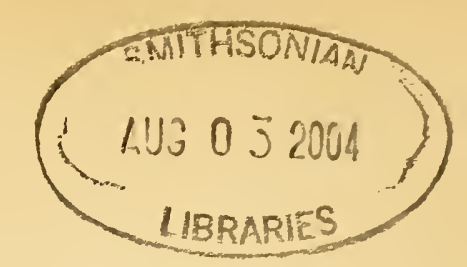

\section{CAUSERIE}

SUR

\section{LE PAYS DES MANGBETTUS}

\section{Messieurs,}

Appelé par le président du Cercle Africain à l'honneur de prendre la parole dans notre rémion d'aujourd'hui, notre intention première a été de vous présenter une relation plus ou moins circonstanciée du voyage que nous avons fait dernièrement au Congo. Mais plusieurs sujets de l'espèce ont déjà été traités avec talent devant les membres de ce Cercle, et ces travaux ayant rapport aux mêmes contrées, présentent inévitablement, abstraction faite de leur partie anecdotique, de nombreuses analogies quant au fond. D'un autre côté, les indigènes des districts d'occupation récente sont encore très imparfaitement connus et, par conséquent, souvent exposés à être méconnus,

Ces motifs nous ont poussé à prendre pour sujet de notre entretien : l.e pays des Mangbettus.

Ne pensez-vous pas, d'ailleurs, que pour nous, qui cherchons à comnaître le territoire congolais et les populations qui l'habitent, il n'existe qu'un seul moyen pratique d'atteindre ce but rapidement et sûrement : 
c'est que chacun de ceux qui ont voyagé là-bas à un titre quelconque vienne nous exposer ce qu'il a vu, nous dépeindre les régions où il a séjourné et les tribus avec lesquelles il a vécu pendant un temps suffisant pour les connaître? Ce dernier point est essentiel pour ne pas tomber dans des erreurs parfois grossières, provenant de ce qu'on a pris pour une généralité une exception due au hasard ou au caprice. C'est là un écueil qu'il faut éviter a tout prix, sans se laisser entraîner par la perspective séduisante de venir, à son retour au pays, armé d'un bagage considérable de remarques curieuses, originales ou bizarres, épater ses contemporains. Le souci du vrai, la crainte de l'erreur sont les premières vertus du voyageur soucieux de iendre féconds ses efforts et ses fatigues.

J'ai été précédé dans la voie que je viens d'indiquer par plusieurs de mes camarades et j'espère que j'y serai suivi par un nombre plus considérable de collègues africains. Lorsqu'on aura ainsi réuni une quantité importante d'observations sùres, d'impressions ressenties, de remarques faites et de renseignements recueillis sur place, il sera possible de comparer tous ces éléments entre eux et. de les coordonner avec les documents matériels que contient le musée ethnographique de l'Etat Indépendant du Congo. On arrivera ainsi à établir les rapports que doirent aroir eus entre elles certaines tribus noires, aujourd'hui fort éloignées les unes des autres, en mettant en lumière leurs points de contact, leurs affinités, la similitude de certaines de leurs coutumes; et l'on parviendra peut-être de cette façon à tirer du chaos l'histoire de ces peuplades intéressantes du centre africain, actuellement encore entourée de ténèbres. Ce sera là un travail long et laborieux, mais qui, mené à bonne fin, présentera incontestablement un intérêt supérieur. 
D'après ce que nous venons de dire de l'histoire de la race noire en général,vous supposez bien que nous n'avons pas la prétention de vous faire celle des Mangbettus en particulier. Il nous parait cependant indispensable de dire quelques mots de l'origine des occupants actuels de la région telle que la tradition l'a transmise aux indigènes qui s'y sont succédé. La population primitive était les A'Bangbas. A une époque que nous ne saurions préciser, mais qui ne peut être considérablement éloignée puisque le temps n'a encore introduit aucune variante dans la relation des évènements qui s'y rapportent, le territoire fut envahi par des bandes nombreuses de guerriers : elles venaient de très loin dans la direction du sud-est, de l'autre côté d'une grande eau, d'après l'expression employée par les indigènes. (Il s'agit probablement ici de l'Unyaro, quoiqu'il n'y ait aucune certitude à cet égard.) Après une lutte acharnée, ces guerriers, qui n'étaient autres que les Mangbettus, s'établirent en maîtres dans le pays. Mais les chefs de race Bangba supportèrent ce joug avec beaucoup l'impatience et ne négligèrent aucune occasion pour essayer de s'en débarrasser. La lutte se prolongea ainsi pendant longtemps, ardente et sourde d'une part, implacable et cruelle de l'autre. Enfin, il y a quelques années à peine, le chef Jangara, le plus important des chefs de dynastie Bangba, infligea une défaite décisive aux partisans du chef Munza (roi des Mangbettus) et à leurs alliés, les MatambaTambas.

Ce fut à ce moment qu'arriva sur le Haut-Uellé la première expédition belge, commandée par le capitainecommandant Van Kerkhove. Peu de temps après, cet éminent officier envoya au gouvernement de l'Etat Indépendant un rapport remarquable, exposant très clairement et très exactement la situation politique de la 
région. Ce rapport a été publié il n'y a pas longtemps par la Belgique coloniale; je vous en cite une page relative à la question qui nous occupe :

" Mal vu de ses voisins, Jangara possède au contraire " l'estime et la confiance des populations nombreuses et

" diverses qui habitent son territoire. Il est réputé comme

" très sage et doué de qualités guerrières. Excellent et

" élégant orateur, il arrive à provoquer successivement

" l'enthousiasme, la stupeur et l'indignation de son audi-

" toire dans les assemblées indigènes. Au moment de

" l'expédition, le pays était en proie à des luttes san-

"glantes de tribu à tribu. C'est ainsi que les bandits de

"Nyangwé et de Kibonghé, arrivés sur les confins de

" ce territoire, y trouvèrent un champ tout préparé pour

$"$ leurs intrigues et leurs odieuses opérations.

"Ils apparurent d'abord sur le Népoko. Les émissaires

" de Munié-Moharra et de Kibonghé reconnurent le pays

" en s'y rendant comme commerçants; ils étudièrent les

"relations entre les chefs et la valeur des alliances qu'ils

* pouraient se créer. S’appuyant tantôt sur les uns,

" tantôt sur les autres, ils les poussaient à des guerres

" dont seuls ils tiraient profit. Toutes ces magnifiques

" contrées devinrent ainsi un théâtre de désolation.

" Munis de butin, d'ivoire et d'esclaves, les Arabes se

" retirèrent pour mettre en sùreté les richesses conquises.

"Ils avaient entendu parler vaguement de l'approche des

" blancs; ils déclaraient qu'ils étaient leurs serviteurs et

" parlaient de leurs bâteaux et de leurs richesses; peut-

" être espéraient-ils exploiter les blancs comme ils l'ont

" fait avec Stanley et bien d'autres; la destruction du

" camp de la Mokongo leur avait appris cependant la

"vérité.

"Avec l'appui d'une bande d'Arabes et d'une multitude

" de Mangbettus, le chef M'Bala pénétra jusque Tangasi 
" et enlera la place. Jangara fut vaincu, mais dans un

" dernier effort il obligea les envahisseurs à se retirer et

" les massacra presque tous dans leur retraite devenue

" une déroute. M'Bala fut tué d'un coup de lance et un

" grand nombre de Mangbettus périrent; peu d'entre

" eux parvinrent à s'enfuir. Ces malheureux alimentèrent

" pendant longtemps encore la cuisine des chefis de

" Jangara Celui-ci avait remis à Milz et à Semio ce qui

" restait de ces bandits, 25 environ. Ils sont presque tous

"Bakumus.

" Jangara respirait, mais une tempête plus terrible le

" menaçait. Il caractérisait la situation en disant lui-

" même : " La fin de l'année sera la fin de mon autorité

" et de mon existence. "Il n'exagérait certes pas. Il était

" sous le coup des entreprises de Uando, son ennemi

" éternel. La puissance de cette famille d'Avunguras

" s'était singulièrement accrue par l'union de tous ses

" membres. Uando s'était retiré vers l'est laissant son

" ancien territoire à deux de ses fils. M'Bittima s'était

" étendu sur les deux rives du Kibali et un autre fils

" s'était installé dans le Makraka où il avait soumis à

" son autorité les Mundus et les Abokas.

"Voilà les ennemis qui allaient fondre sur Jangara du

" côté de l'est et du nord ; Munza devait agir à l'ouest,

" avec l'aide du blanc, pensait-il ; Assanga, M'Belia et

" d'autres chefs devaient l'attaquer par le sud. La con-

" centration des forces ennemies était faite, quand Milz

" arriva chez Maï-Munza.

"Jangara lui-même avait réuni ses troupes dans la

" Zériba de la Gadda. L'heure de la lutte était proche.

" La famille de N'Dula ne comptait plus son existence

" que par heures.

" On peut dire, à ce propos, que le rôle de l'expédition

" fut providentiel. Le moment était venu de préserver 
" de l'invasion arabe l'immense contrée des A' Bandja;

* elle rompit les négociations qui s'étaient engagées entre

" les Sassa et les Arabes ; elle préserva de nourelles

" invasions les territoires sitnés sur la rive gauche de

" l'Uellé. Son arrivée fit replier les arabes sur le Népoko

" et plus loin encore; ils abandonnaient ainsi la province

" des Mangbettus et les territoires de Bauli et de Kanna.

" Milz et Semio parvinrent à dissoudre la coalition qui

. s'était formée contre Jangara et à renvoyer les troupes

" de Uando, campées à quelques journées en amont. Bien

" plus, peu de jours après, tous les chefs de cette coalition

" firent leur soumission à l'État et lui offrirent leurs

* services. Ce fut l'œurre de Milz. Aux yeux du comman-

" dant Van Kerckhoven, elle suffisait à faire ressortir

* tout le mérite de cet officier. Le pays était rentré dans

: une période de tranquilité.

Cette tranquilité n’a plus guère été troublée d'une façon sérieuse depuis lors, quoique le territoire Mangbettu ait été menacé vers la fin de 1894 d'une invasion mahdiste. L'expédition de ces derniers, conduite par l'émir El Têr, fut victorieusement repoussée par les troupes de l'État sous les ordres du commandant Francqui.

$$
\text { *** } *
$$

Jetons maintenant un coup d'œil sur l'aspect gónéral de la région :

Le pays des Nangbettus est limité an nord par l'UëlléMakua; au sud par le Bomokandi; il confine à l'est aux territoires des populations Monfus avec une frontière assez ragucment déterminée; rers l'ouest il s'étend jusqu'au pays des A' Barambos et à pour limite un petit affluent de l'Uëllé, la Kieliwa.

Abstraction faite de quelques hauteurs isolécs, la région ue présente que des ondulations très peu prononcées. Une 
ligne de collines, la traverse sensiblement de l'est à l'ouest, à peu près à égale distance du Bomokandi ct de l'Uellé : c'est la ligne de partage des bassins de ces deux rivières. A cette crète viennent se rattacher des collines aussi nombreuses que peu importantes, dessinant les vallons des innombrables petits ruisseaux tributaires des deux rivières principales; elles sont toutes parrallèles entre elles et se dirigent du sud-est au nord-ouest sur le versant de l'Uëllé et du nord-est vers le sud-ouest sur celui du Bomoliandi.

Dans la partie ouest du pays ainsi que dans la vallée du Bomokandi, les forêts sont nombreuses et composées d'arbres d'essences très variées, parmi lesquelles on remarque le bambou et le bois rouge. Elles y alternent arec des espaces considérables couverts d'herbes atteignant parfois 3 mètres de hauteur. Dans la partie orientale ces dernières dominent, et l'aspect monotone de la contrée n'est releré que de quelques bandes boisées suivant les rives des cours d'eau. Par suite de la composition argileuse du sol et de son degré d'imperméabilité plus ou moins prononcé, selon les endroits, par suite, aussi de la pente très-faible des deux versants principaux, les marécages sont assez fréquents, surtout pendant la seconde moitié de l'année (saison des pluies). Ils constituent, avec les hautes herbes, les principaux obstacles à la viabilité de la région.

Le sol est d'une très grande fertilité : pas un pouce de terrain qui ne soit couvert de forêts aux arbres gigantesques, de riches plantations indigènes de bananiers, de maïs, de sorgho, d'éleusine, de noix d'arachides, etc., ou encore, d'une luxuriante et sauvagre végétation d'herbes et de lianes. Chaque poste de l'Etat a son potager produisant la plupart de nos légumes d'Europe, à condition de protéger les jeunes plants contre les ardeurs trop 
violentes du soleil : voilà pour ce qui concerne le bienêtre matériel de l'indigène ct de l'européen.

Nais ici se pose tout naturellement la question de savoir si la région est susceptible de derenir, pour l'exploitant, pour le commerçant, une source de richesse. Nous n'en doutons pas, en basant notre opinion sur les considérations suivantes : les chefs possèdent pour la plupart de grandes quantités d'ivoire; celles-ci ont été réunies petit à petit parce qu'ils en connaissent la valeur relative depuis très longtemps, à cause de leurs relations arec les Egyptiens; mais nous n'en parlerons que pour mémoire, admettant que le rendement de cette matière est destiné à subir ume diminution progressive. Nous derons cependant faire remarquer que les Arabes n'ont pas eu le temps d'organiser au pays Mangbettus, ces immenses hécatombes d'éléphants, n'ayant en vue que le bénéfice immédiat: que par suite, ces pachydermes sont encore très nombreux dans cette région, surtout à l'est, et que d'ici à de longues années, leur race n'est certainement pas menacée d'extinction.

La terre, dans son inépuisable fécondité offre des éléments d'appréciation plus importants et plus stables, la liane à caoutchouc, ce précieux produit de plus en plus recherché sur nos marchés, est amplement représentée dans la région par la plupart de ses variétés. Pour donner une idée de son abondance dans la contrée, je citerai ce seul fait, que la zériba (1) de la station de Nyangara, ayant à peu près 500 mètres de périmètre, est composée presqu'exclusivement d'une essence donnant du latex; or, les «sticks " qui ont servi à la construire ont été coupés au hasard, sans faire aucune sélection. Ils ont presque tous pris racine et entourent actuellement les

(1) Espèce de palissade construite en branches d'arbres juxtaposées. 
bâtiments de la station, d'une immense muraille de verdure du plus riant aspect.

D'un autre côté, les essais de culture du caféier, faits au moyen de graines envoyées de la station de l'Équateur ont donné les meilleurs résultats ; ces essais ont produit au bout d'un an 1,500 plantes très-vigoureuses ayant en moyenne 80 centimètres de hauteur.

Et qui pourrait dire quelles sont à côté des ressources naturelles et certaines que je viens de citer, celles que nous réserve une connaissance plus complète du pays, notamment en ce qui concerne le sous-sol, encore complètement inexploré et totalement ignoré?

Nous n'hésitons donc pas à répondre aflỉrmativement à la question que nous nous sommes posée, sans craindre d'être démentis par les érènements; et, à ceux qui nous objectent que des prévisions et des espérances ne suffisent pas dans la matière, nous répondrons que l'obstination à ne pas rouloir suirre les déductions amenées par le raisonnement suffirait encore moins en sens contraire et pour pouroir exiger la preure par le fait accompli il faut aroir la patience d'attendre que les choses aient pu suivre leur cours naturel.

Nous allons maintenant essayer d'exposer brièvement quelques observations destinées à guider notre appréciation sur les indigènes. J'ai peine à résister à la tentation qui me poursuit d'aborder ce sujet par une phrase que je me souvièns avoir lue et avoir entendue différentes fois déjà, la voici : "La race Mangbettu (ou une autre, celle dont on parle) est sans contredit une des plus belles parmi celles qui peuplent l'Afrique ". Mais, à quelques très rares exceptions près, toutes les tribus noires présentent des types remarquables de très beaux spécimens de la race nègre; et je pense qu'en établissant entre elles une comparaison conduite sans idées préconçues, un 
classement quelconque serait difficile à faire. Nous nous bornerons donc à dire que le Manglbettu eśt généralement de haute taille, bien conformé et bien proportionné. L'aspect de sa robuste charpente, donnant attache à une musculature souple et solide qui se révèle à la surface en reliefs puissants nettement et harmonieusement bosselés, fait naître irrésistiblement une impression de vigueur virile et de force corporelle. Comme dans beaucoup d'autres tribus, l'éducation physique commence dès la plus tendre enfance, on pourrait dire au berceau, si cet instrument de supplice n'était totalement inconnu là-bas. Il est rare de traverser un village Mangbettu sans voir des marmots pas plus haut que ça, s'exerçant à jeter des roseaux, qui, dans leur imagination enfantine, représentent des sagaies et des lances. Cette éducation fait du Mangbettu adulte, un guerrier hardi et redoutable, un chasseur intrépide et adroit et un infatigable marcheur.

Quant au moral, le Mangbettu n'est pas ce sauvage stupidement et systématiquement hostile à tout ce qui est étranger à sa tribu! Ce sauvage, que d'ici on se figure volontiers devoir être de plus en plus intraitable de plus en plus farouche, à mesure qu'on s'avance dans l'intérieur de l'Afrique! Beaucoup s'en faut, et nous avons eu plusieurs fois la satisfaction de constater qu'il n'est pas inaccessible à certains bons sentiments, et même à certaines vertus. Il suffira pour le montrer de rappeler l'aventure de Miani, notre célèbre et malheureux prédécesseur dans cette contrée : abandonné par la caravane arabe qui l'avait amené à Tangasi, après qu'elle lui eut extorqué tout ce qu'il possédait, il vécut au milieu des peuplades mangbettus pendant plusieurs années, sans avoir rien à leur donner, et sans cependant avoir à souffrir de leur part la moindre vexation, comme l'attestent ses notes de voyage. Après sa mort, ils lui donnèrent la 
sépulture et eurent pour lui une attention plus touchante encore que naïve : comme il était grand fumeur, ils jugèrent qu'il lui serait pénible de renoncer du jour au lendemain à son habitude favorite, et ils placèrent dans sa tombe, à portée de sa main, une pipe et une petite provision de tabac.

L'indigène montre arec complaisance, à l'Européen passant dans les environs de Tangasi, le petit monticule, aujourd'hui envahi par les herbes, où fut enterré l'infortuné explorateur italien. Et l'empressement, le sentiment d'amour-propre risible avec lequel il conduit le blanc à ce pieux pélerinage, montre à l'évidence qu'il se rend compte de la noblesse et de la générosité de l'action accomplie par ses frères.

Faut-il encore citer l'exemple fourni par les voyages de Junker? Escorté par Semio, Sultan Sandeh, jusqu'à la rive de l'Uellé, à hauteur de Mambanga, il traversa, après le départ de son guide, tout le pays Mangbettu; il n'était accompagné que de quelques serviteurs armés. On peut dire, il est vrai, qu'il arait pour lui le prestige de la puissance de Semio et la protection du gouvernement égyptien.

Loin de nous, d'ailleurs, l'intention de faire croire que le Mangbettu ne cherchera pas à tirer de l'hospitalité qu'il accorde, tous les profits et tous les avantages qu'elle est susceptible de lui procurer. Mais le désintéressement est-il donc si fréquent chez nous que nous ayons le droit de faire un grief au noir de son goùt prononcé pour le gain?

Les femmes Mangbettu sont généralement assez bien douées par la nature au point de vue physique. Beaucoup d'entre elles ont des traits assez réguliers, la physionomie sympathique et la gorge aux fermes appas est sourent gracieusemeut modelée; ajoutez à cela une taille hardi- 
ment cambrée, des membres aux lignes élégantes, aux extrémités petites et fines, aux attaches félinement souples et flexibles; vous aurez ainsi le portrait de lia Vénus mangbettu, à la démarche empreinte d'une prorocante coquetterie. Mais, si vous ètes quelque peu sentimental et que vous craigniez l'atteinte glaciale de la désillusion, gardez-vous bien de pousser plus loin votre admiration! Car vous apprendriez bientôt que cette enveloppe aux multiples séductions courre une âme vibrant difficilement aux accents de l'amour. Hâtons-nous de dire, cependant, que cette réserve, cette froide impassibilité n'est qu'apparente, car les femmes Mangbettus que l'on rencontre ont généralement un enfant par la main, un nourrisson attaché à la hanche au moyen d'une lanière en écorce et un autre... en expectative.

Aucune de ces particularités n'est d'ailleurs exclusive à la femme Mangbettu; mais ce qui frappe tous ceux qui voyagent dans ce pays, c'est la place qu'elle occupe dans l'existence de l'indigène et l'importance du rôle qu'elle remplit dans la société. Quelle différence sensible entre sa condition sociale et celle des femmes de la plupart des tribus du Congo, où la femme tient le milieu entre l'animal et l'être humain, où elle n'est généralement considérée que comme une machine à défricher le sol et à assurer la continuation de la race!

Que l'Européen observe, par exemple, ce qui se passe à son arrivée dans un village Sandeh (1). Les femmes auront été mises soigneusement à l'écart, afin de les soustraire aux regards des soldats de l'escorte et à leurs entreprises parfois impertinentes, mais surtout parce que la femme est, aux yeux de ces indigènes, un être absolument inférieur, n'ayant rien à voir et du reste incapable de rien

(1) Il s'agit ici des A'Sandeh riverains de l'Uellé, car l'immense race des A'Sardeh, comprend également, surtout rers le nord. des tribus très avancèes. 
comprendre à l'entrevue qui va avoir lieu. Si le hasard rous fait rencontrer une de ces malheureuses et que vous lui adressiez la parole, consciente de son infériorité, de sa nullité vis-à-vis de cet être supérieur qu'elle a devant elle, elle n'osera pas articuler un mot; et si,à force d'insistance, vous parvenez à obtenir une réponse, celle-ci sera faite d'un ton si timide et si bas, qu'il vous sera impossible d'en saisir même le sens.

Comparons cela à ce qui se passe, dans des circonstances analogues, chez les Mangbettus. L'Européen y est reçu, sous le hangar établi à cet effet au centre du village, par le chef entouré de quelques notables, ses conseillers, et de ses principales femmes. Celles-ci, beaucoup moins farouches que leurs sœurs A'Sandeh, loin d'éviter le regard de l'homme blanc, sont très flattées de s'apercevoir qu'elles attirent son attention et ne dédaignent pas, loin de là, de faire avec lui un bout de conversation. Elles assistent à la palabre, reçoivent des présents et en font en leur nom personnel et, parfois, prennent une part active à la discussion. Et, lorsqu'il s'agit d'arranger un différend, d'aplanir une difficulté ou de conclure une convention, ces orateurs du beau sexe ne sont pas toujours les moins éloquents, ni ceux dont les arguments sont les plus faciles à combattre.

L'ancien chef des Mangbettus, le sultan Jangara, était ce que nous appellerions ici un féministe convaincu : il est de notoriété qu'il ne prenait aucune décision importante sans avoir consulté sa principale femme, Neusima, qui, pendant de longues années, a exercé dans la tribu une influence considérable et absolument incontestée.

Ce portrait serait incomplet si je n'y joignais une description succincte de l'accoutrement usité chez les Mangbettus :

L'homme porte généralement le pagne en étoffe d'écorce. 
Les gens " chic " le portent très ample, le pli bien fourni et artistement drapé descendant jusqu'au-dessous du genou; les deux extrémités sont fortement relevées et affectent la forme de grands éventails courrant presque complètement la poitrine et le dos. Il n'y a guère que les chefs qui, dans des circonstances particulières, par exemple pour faire une visite cérémonieuse dans une station de l'Etat ou pour receroir un blanc occupant un rang élevé, s'habillent à la façon arabe : pantalon bouffant et chemise longue, serrée à la taille, en tissu de coton indigène ou en americani (1). Un fez en feutre amarante et des pantoufies en cuir d'antilope complètent parfois ce costume.

La toilette de la femme est d'une simplicité qui en complique singulièrement la description. Elle se compose d'un petit, très petit tablier rectangulaire, dont la plus grande dimension ne dépasse parfois pas 10 centimètres; ce petit tablier, transformation éridente de l'antique feuille de vigne, a pour symétrique, du côté du dos, une moderne feuille de lotus. Cette seconde partie du costume présente comme particularité déconcertante qu'elle n'est fixée aux reins par aucune agrafe, ni par aucun lien artificiel, tous ceux qui constatent ce détail pour la première fois en restent terriblement intrigués. Mais, en y regardant de plus près, le mystère ne tarde pas à s'éclaircir ; et l'on ne peut s'empècher d'admirer la nature, qui, dans sa préroyante sagesse, donna aux feuilles de lotus une tige longue et lisse et dota en même temps, la femme Mangbettu, de formes au galbe opulent, exerçant l'une sur l'autre une compression naturelle amplement suffisante pour maintenir en place un appareil bien plus lourd.

Les individus des deux sexes ont un soin extrême de leur coiffure originale et caractéristique : une cordelette

(1) Tissu de coton écru de fubrication euroṕenne. 
en fibres, teinte en noir, très longue et de l'épaisseur d'une mêche de fouet, part du front à environ deux doigts au-dessus des yeux, passe derrière les oreilles et s'enroule autour du crâne à la façon dont le fil de nos tailleurs s'enroule sur sa bobine. Les différents tours sont soigneusement juxtaposés, sans le moindre intervalle, et ne laissent passer l'extrémité d'aucun cheveu; ces derniers, que les Nangbettus portent longs, contrairement à l'usage généralement répandu au Congo, sont ainsi ramenés vers le sommet de la tête, où ils se réunissent en un chignon monumental surmonté d'un minuscule bonnet en paille tressée, sans bords et à fond carré. Parfois ce couvre-chef est orné d'une houppe en plumes de pintade, ou en plumes rouges fournies par la queue du perroquet si commun en Afrique. L'usage de ces plumes rouges constituent une espèce de privilège réservé aux gens de race. L’ensemble de l'édifice pileux décrit ci-dessus est enjolivé et consolidé au moyen de longues épingles en ivoire, en cuivre, en fer ou en bois. Plus il est allongé, plus on le troure beau. Aussi voit-on les mères mangbettus comprimer la tète de leurs nouveaux-nés au moyen de minces bandelettes d'écorce, dans le but de lui donner la forme la plus allongée possible vers l'arrière, et d'assurer ainsi à leurs rejetons, devenus adultes, un avantage physique hautement apprécié du beau sexe.

Le tatonage de la figure n'est pas fort en honneur chez les Mangbettus ; ils se rattrappent largement, sous ce rapport, sur la poitrine, le dos et le haut des bras. Il se compose presque invariablement de lignes pointillées parallèles, ou de courbes concentriques suivant le contours des muscles. Les élégants, et surtout les élégantes, combinent ces atours peu encombrants, solides et pas coùteux, arec des bariolages obtenus par le suc du fruit du gardenia; ce dernier fournit une teinture d'un beau noir, tranchant 
nettement sur la couleur beaucoup moins sombre de l'épiderme mangbettu. C'est encore là un de ces usages presque généralement répandu dans tout le Congo. Seulement chez la plupart des tribus, l'ornementation dont nous parlons est un barbouillage informe composé de points, de lignes, de tâches irrégulières, de ronds, de plaques d'une certaine étendue, et ces différentes maculations faites au hasard ne présente à l'œil aucun ensemble quelconque.

La femme Mangbettu, au contraire, se confie au talent d'un spécialiste du genre, qui avec une patience et un art dignes d'une cuvre plus durable, étant donné que cette teinture ne tient que pendant deux ou trois jours, produit de véritables dessins parfois très jolis et toujours très réguliers. Leur variété ne connait pas do limites, tout y passe : le filet du pêcheur comme le pince-nez du blanc, les mouchetures du léopard et les stries du zèbre comme les carrelages les plus réguliers de nos mouchoirs et les arabesques les plus compliqués de nos cotonnettes d'échange.

Si nous relevons ce détail, qui peut paraître insignifiant à première rue, c'est qu'à nos yeux il constitue un indice sérieux de la facilité avec laquelle le Mangbettu s'assimile, les choses qui, jusqu'ici, lui ont été inconnues, et une preuve de ses tendances et de ses dispositions artistiques : ce sont là, nous semble-t-il, deux facteurs puissants pour élever rapidement un peuple relativement primitif, à un degré de civilisation supérieur.

L'organisation sociale des Mangbettus est évidemment très simple. A la tête de la tribu se trouve le grand chef ou sultan (ce mot est un dérivé du mot arabe " soultân " qui signifie chef ; il ne faut pas lui donner la signification que nous y attachons en Europe). Viennent encore un nombre considérable de chefs secondaires d'importance 
très variable presque tous parents ou alliés du chef principal. Autour de ceux-ci se groupent les guerriers, vivants comme ils l'entendent et astreints envers leur chef direct à la seule obligation de le suivre à la guerre ou à la chasse.

Les chefs ou les guerriers possèdent, suivant leur degré de richesse, un nombre plus ou moins considérable de femmes et d'esclaves. Ce dernier mot est très improprement employé : ces esclares ne sont en réalité que des serviteurs à vie; leur existence est identique à celle do leur maître, sauf évidemment, qu'ils ne jouissent pas de la même considération. Ils ont généralement une femme et une famille, mais leur chef dispose comme il l'entend de leurs rejetons.

Le commerce d'esclares, la traite, n'a jamais existé chez les Mangbettus que d'une façon passagère, accidentelle et sous la pression d'une influence étrangère. A l'époque où leur territoire faisait partie de la prorince égyptienne du Guruguru, notamment, elle s'est manifestée sous un aspect particulièrement odieux : à l'insu du gouvernement égyptien, toujours très peu au courant de ce qui se passait dans ces provinces éloignées, des fonctionnaires aussi astucieux que haut placés forçaient les petits chefs indigènes ì leur livrer un certain nombre de jeunes garçons, moyennant une faible somme d'ailleurs très irrégulièrement payée. Ils faisaient subir à ces malheureux une mutilation horrible, pratiquée généralement arec une maladresse et une brutalité féroces, et vendaient à prix d'or, aux harems de l'Orient, ceux que leur robuste constitution sauvait du trépas. Mais cet infâme trafic répugnait profondément aux Mangbettus et disparut arec les moudirs égyptiens; longtemps encore cependant il laissa des traces dans le pays, car parmi les rictimes de cette honteuse spéculation, un assez grand nombre de jeunes gens, ne se sentant sans doute pas la rocation 
nécessaire, pour aller au loin garder la vertu des houris, parrenaient à s'échapper en cours de route, et cahin cala, revenaient au pays. C'est ainsi que Juncker, lor's de son passage chez les Mangbettus, il y a une douzaine d'années à peine, avait à son service deux jeunes garçons, ou plutôt deux jeunes Mangbettus, qui après avoir subi l'opération qui devait les rendre aptes à remplir les fonctions auxquelles on voulait les destiner; avaient réussi à prendre la fuite et avaient été recueillis par le célèbre voyageur.

L'esclavage, comme on l'entend généralement, n'existe donc pas chez les Mangbettus. Jamais nous n'arons ru un chef vendre ou proposer la vente d'un lomme ou d'une femme de sa tribu quelle que fut leur position sociale; ils ont fourni il est vrai, un certain nombre d'esclares aux Egyptiens et aux Natamba-Tambas, mais c'étaient des prisonniers de guerre capturés dans les combats livrés aux populations voisines, sur les instigations de ces arabes.

Il suffira d'ailleurs de dire, pour caractériser le genre. d'esclarage qui existe chez les Manghettus, qu'il n'est pas rare de voir un de ces soi-disant esclares, croyant avoir à se plaindre de son maître, profiter de la première occasion venue pour le quitter et pour aller se mettre sous les ordres d'un chef voisin, où il est généralement le bienvenu.

Passons à un autre ordre d'idées et analysons le Mangbettu au point de vue de ses croyances et de ses pratiques religieuses. Les observations de l'espèce sont fort intéressantes à faire, car elles permettent parfois d'établir des ressemblances curieuses, indices de la communauté d'origine de tribus qui sont derenues fort différentes les unes des autres.

La destinée de l'indigène Mangbettu est entre les mains 
d'un être mystérieux, auquel son imagination n’assigne aucune forme déterminée. Résidant dans les plus grandes profondeurs de la Makua, à un endroit ignoré de tous, il abandonne parfois cette retraite pour s'incarner tantôt dans le corps d'un léopard, tantôt dans celui d'un crocodile ou d'un serpent, tantôt dans celui d'un autre animal malfaisant. Jamais il ne se dérange que dans le but de faire du mal, et malheur à celui qui se trouverait sur son chemin. Tous les Mangbettus sont convaincus de son existence et de son pouvoir néfaste quoique aucun d'eux ne l'ait jamais ru, car celui qui oserait le regarder mourrait aussitôt. Lor'squ'un individu meurt, c'est auprès de cet être surnaturel qu'il se rend, par un chemin que nul ne connaît et que lui-même oublie, dès qu'il l'a parcouru.

Remarquons en passant que ce ne sont déjà plus là des croyances barbares et primitives n'admettant que des divinités toutes matérielles, comme c'est encore le cas, pour beaucoup de tribus noires. Elles permettent de conclure, sinon que les Mangbettus croient à l'existence de l'âme et à son immortalité, du moins qu'ils admettent une existence future faisant suite à leur vie terrestre.

Les indigènes dont nous nous occupons n'ont, à proprement parler, pas de fétiches, à moins qu'on ne veuille donner ce nom à des amulettes de forme et de nature variées, espèces de talismans, ayant chacun ses propriétés et ses vertus particulières : une dent de léopard donnera la force et le courage, la queue du chat sauvage assurera la victoire dans le combat, les graines de l'accacia, réunies en bracelet, procureront le succès dans les expéditions amoureuses, ct ainsi de suite.

Si les Mangbettus n'ont pas de fétiches, ils sont en reranche presque tous féticheurs. Les féticheurs officiels ont pour mission de faire les conjurations nécessaires pour 
pénétrer les secrets de l'avenir, lorsqu'il s'agit de questions importantes d'intérêt général. Les marques extérieures de ces cérémonies consistent invariablement en chants énergiquement rhytmés, accompagnés de danses échevelées et de contorsions étranges. Il est à remarquer que le féticheur ne possède pas ici cet ascendant redoutable qui, dans beaucoup de tribus, met entre ses mains le sort de chacun; car, lorsque la réponse de l'oracle n'est pas conforme à ses intentions, le chef Nangbettu n'hésite pas à décider que le féticheur a mal opéré et il fait recommencer la cérémonie.

Il y a trois manières différentes pour se renseigner sur l'issue favorable ou funeste d'une entreprise, ou sur la culpabilité ou l'innocence d'un accusé. La plus curieuse et la seule, d'ailleurs, qui soit pratiquée presque exclusivement par les Mangbettus et les tribus qui les environnent, est le " Mapinghé ": dans une case généralement assez vaste, pour contenir une assistance nombreuse, très rarement en plein air ou sous un hangar, on dispose horizontalement un ou plusieurs trones de bananiers; dépouillés de leurs feuilles extérieures, ils ont l'aspect de cylindres parfaitement lisses; leurs extrémités reposent sur des piquets fichés dans le sol et se recroisant en forme d'X, suivant la génératrice supérieure de ces cylindres et perpendiculairement à celle-ci, on dispose, en équilibre, un nombre considérable de petites baguettes également cylindriques, en bois dur, poli et huilé. Elles sont réunies en séries de trois, se touchant dans toute leur longueur; deux d'entre elles sont en contact avec le bananier, la troisième délicatement posée sur les deux premières. L'ordre suivant lequel ces petits bâtonnets sacrés doivent être placés pour se conformer aux rites traditionnels, est indiqué au moyen de petits cercles en fer, incrustés dans leurs bases, en nombre variant de un à cinq et différem- 
ment disposés. On comprend facilement qu'il faut un rien pour que l'équilibre du système soit rompu et que les bâtonnets tombent sur le sol. Or, c'est précisément le temps plus ou moins long, pendant lequel l'équilibre persiste et le nombre de baguettes qui tombent de part et d'autre du tronc de bananier qui constituent les éléments d'interprétation de la réponse favorable ou défavorable du Mapinghé. Ces circonstances se produisent, d'ailleurs, entièrement suivant la volonté du féticheur. La grande habitude qu'il a d'arranger le dispositif décrit, son talent d'équilibriste, lui permet de placer les petites pyramides de trois bàtonnets, de manière que leur centre de gravité se trouve, tant soit peu, à droite ou à gauche de la ligne de sustentation, mais pas d'une quantité suffisante pour provoquer immédiatement leur chute : celle-ci se produira naturellement du côté du centre de gravité. D'autre part, le féticheur provoque cette chute, au moment précis où il le désire : pendant toute la durée de la cérémonie, on le roit courbé en deux, battant des mains, longer le bananier-oracle en exécutant des danses sacrées, cadencées par le chant de tous les assistants. Léger comme une plume, il effleure à peine le sol sur lequel il se livre à ses exercices sacro-chorégraphiques; mais lorsqu'il juge le moment venu, d'un lourd appel du pied exécuté à l'une des extrémités, à l'endroit même où se trouvent enfoncés les piquets de soutien, il communique à ceux-ci une légère trépidation qui provoque l'effondrement. Le Mapinghé a parlé et il ne reste plus qu’à interpréter la décision qui lui avait été dictée.

Une pratique plus répandue que la précédente, probablement parce qu'elle est à la portée de tout le monde, est celle du " Benghé $r$. Elle est très répandue également chez les populations A'Sandeh. Il y a deux manières de " faire Benghé ". La première consiste tout simplement 
à prendre un certain nombre de poules au plumage blanc et à les jeter toutes ensemble dans la rivière. Survant le nombre de rolatiles qui se noient, c'est-à-dire, suivant le tribut que prélève la mystérieuse divinité, dont nous avons parlé, elle fait connaître si elle favorisera ou non le projet de celui qui la consulte.

La seconde méthode, un peu plus compliquée, est une espèce d'épreure du poison appliquée à une ou à plusieurs poules. Nous avons eu l'occasion d'observer de très près la cérémonie, qui, dans une campagne contre le chef Sandeh M'Biri, où nous étions accompagnés d'une troupe d'auxiliaires Mangbettus, se renouvelait régulièrement toutes les nuits.

Au moyen de petites herbes, semblables à l'herbe commune de nos prairies, on forme une botte de la longueur et de la grosseur du doigt. Tous les gros bouts des brins sont soigneusement placés du mème côté et fortement serrés les uns sur les autres, au moyen d'une ligature. La petite botte ainsi formée, est trempée dans une décoction de plantes vénéneuses et placée ensuite dans le pli d'une feuille préalablement découpée en forme d'ovale, très allongée et pliée suivant le grand axe de l'ellipse. Elle est ainsi complètement cachée aux regards des assistants et il n'y a plus que l'opérateur qui, par une légère pression des doigts, puisse se rendre compte du côté vers lequel se trouve le gros bout de la botte. Or, s'il administre le poison à la poule par cette extrémité, les herbes comprimées les unes sur les autres, forment bouchon, la quantité de poison qui s'écoulera sera très minime et la poule en réchappera neuf fois sur dix. Ce sera tout le contraire, s’il administre le Benghé par l'extrémité opposée.

Ce qui est curieux à noter, c'est que tout le monde connaît ces trucs, tout le monde les applique à l'occasion, 
et personne n'entreprendra rien sans avoir " fait Benghé " une ou plusieurs fois : tellement est grand, chez le noir comme chez nous, le besoin d'espérer quand même, quelque fragile que soit la base qu'on peut donner à ses espérances.

A la question des croyances religieuses, se rattache tout naturellement celle des cérémonies funèbres. Malheureusement nous n'arons pu nous procurer sur ces dernières des détails typiques, en ce qui concerne les Mangbettus. Chaque fois que nous amenions la conversation sur ce sujet, nous n'arons obtenu que des indications g’énérales, ne nous rérélant rien de bien nouveau; à la mort d'un chef ou d'un personnage occupant dans la tribu une situation élevée, les habitants du village et les amis et connaissances de dix ou quinze lieues à la ronde, se réunissent pour célébrer les funérailles. Pendant plusieurs jours, parfois pendant plusieurs semaines, ce sont des chants et des danses interminables, accompagnés d'abondantes libations de " Merissa ", bière indigène de maïs ou de sorgho.

La chair humaine entre-t-elle dans la composition de ces macahres agapes? Nous ne pouvons le certifier, car jumais nous n'avons constaté, de façon certaine, un cas de cannibalisme. Mais il y a de fortes présomptions de croire qu'il en est ainsi. Il serait extraordinaire, en effet, que seuls au milieu d'une immense population de peuplades anthropophages, les Mangbettus formerait un groupe distinct et isolé, répudiant une pratique presque partout répandue. Mais le soin qu'ils mettent à se cacher lorsqu'ils s'y abandonnent, l'indignation, tout au moins apparente, arec laquelle ils répondent à celui qui les interroge à ce sujet, sont des indices qui permettent d'espérer que ce vice répugnant deriendra de plus en plus rare. Sans doute, plusieurs générations se succèderont 
encore arant qu'il n'ait complètement disparu ; mais en Afrique les générations vont relativement vite et l'on doit espérer que l'influence de l'européen, secondée par l'action du temps, changera finalement sous ce rapport, comme sous beaucoup d'autres, les mours des indigènes.

Les morts sont oints au moyen d'une mixture, composée de poudre de bois rouge triturée dans de l'huile, roulés dansleurs étofies es plusprécieuses et enterrés avec leurs lances et leur bouclier. Chaque fois que nous avons roulu savoir si ces cérémonies sont accompagnées de sacrifices lumains, nous avons obtenu une réponse invariable : " Je ne sais pas, lorsqu'un chef meurt, je me sauve dans la brousse $»$. Mais cette dernière partie de la réponse et le malaise que produit toujours la question, nous fait pencher pour l'affirmative. Une coutume à signaler, c'est que lorsqu'un décès se produit dans la famille d'un chef, la politesse exige qu'on lui adresse des compliments de condoléance, mais sous une forme appropriée au caractère du noir en général: on envoie au chef de la tribu éprourée, un cadeau d'une importance proportionnée au rang qu'on occupe soi-même; et dans ce cas, la récipro-. cité n'est pas de rigueur.

Il nous reste encore maintenant à dire quelques mots des diverses industries du pays Mangbettu.

L'industrie qui, chez les Nangbettus comme chez toutes les peuplades indigènes de l'Uellé, occupe la première place est la métallurgie du fer. Celui-ci se trouve dans toute la partie septentrionale du Congo, en quantité considérable; on y rencontre fréquemment des couches d'argile ferrugineuse ayant une très grande épaisseur. Ce minerai, très riche en fer, puisqu'il se laisse réduire par des moyens absoluments primitifs, se trouve souvent à fleur du sol et ne présente ainsi aucune difficulté à l'exploitation. On le transforme en fer dans des fourneaux 
dont l'usage est d'ailleurs répandu presque partout sauf chez quelques peuplades telles que les Djur et les Bongos; à une espèce de creuset en argile, établi sur le terrain naturel et chargé d'un mélange de minerai concassé et de charbon de bois, aboutissent les tuyaux de trois ou quatre soufflets de forge indigènes; ces derniers sont composés d'un corps en bois ayant la forme d'une grande pipe de fumeur, dont la tête présenterait deux fourneaux distincts en communication avec un tuyau unique, prolongé du côté du foyer par une extrémité en argile. A chacun des deux compartiments de la partic supérieure de cet appareil est adapté hermétiquement une poche en peau de chèrre pouvant se soulever au moyen d'une baguette attachće à sa partie centrale. Lorsqu'on soulère la baguette la poche se remplit naturellement d'air, tandis que par le mouvement inverse l'air emmagasiné est violemment expulsé par le tuyau; par suite d'un jeu rapide et non interrompu des baguettes on parrient ainsi à injecter dans le foycr un courant d'air assez fort. Une partie du fer contenu dans le minerai entre ainsi en fusion et vient s'accumuler au fond du creuset sous forme d'une loupe contenant beaucoup de scories et d'impuretés. On se débarrasse de ces résidus par une série de martelages, et on obtient finalement un métal assez pur et d'une dureté très suffisante.

Chaque agglomération de villages a son forgeron. Ce métier domne à celui qui l'exerce une grande considération dans la tribu et se transmet presque toujours de père en fils. Contrairement à ce que l'on voit chez les populations riveraines de l'Uellé et de l'Oubanghi, les forgerons ne sont pas établis dans les rilages. Ils vivent plus ou moins à l'écart et il nous est même arrivé de trouver un fourneau de forge en plein brousse, loin de toute liabitation. 
L'outillage du forgeron Mangbettu est excessivement restreint.

Une masse prismatique en fer, haute de 20 à 25 centimètres et dont les bases ont 7 ou 8 centimètres de diamètre, enfoncée verticalement dans le sol, sert d'enclume; le marteau est obtenu au moyen d'un lingot de fer, encastré dans un manche en bois fendu en fourche à une de ses extrémités; les deux branches de la fourche, refermées sur le lingot, sont solidement maintenues par une forte ligature en lianes. Outre l'enclume et le marteau, le forgeron indigène ne se sert que du burin, soit pour débiter le métal, soit pour faire sur le fer des armes, des ornements et des dessins imitant la gravure. Depuis que nous sommes établis dans le pays il y a chez l'artisan noir une tendance à compléter son outillage si primitif. Les indigènes sont notamment très contents quand on leur procure des limes et des forets de notre fabrication.

La cuivre se travaille également dans la légrion des Mangbettus, bien que jusqu'ici on n'y ait pas constaté la présence de minerais assez richés pour pouvoir être réduits au moyen du procédé rudimentaire décrit ci-dessus. Les indigènes se procurent ce métal par l'intermédiaire de leurs voisins du nord, qui se fournissent eux-mêmes auprès des N'Sakkaras et auprès des exploitants arabes d'Hofrah-en-Nahâs. Nous leur en fournissons également une assez grande quantité sous forme de fil étiré. (Mitakos). Il sert principalement à forger des bracelets, des anneaux d'oreilles, et surtout des armes de luxe, parmi lesquelles on trouve des objets remarquables.

L'industrie du bois est arrivée, chez les Mangbettus à un degré déjà assez avancé. Les travaux les plus importants comme dimensions sont les pirogues. Elles sont creusées d'une pièce dans le tronc d'un arbre, comme partout ailleurs du Congo; leurs dimensions atteignent 
parfois 10 à 12 mètres en longueur et 1 mètre à 1 mètre 25 centimètres en largeur. Ces embarcations sont génćralement très solides et très bien équilibrẻes.

Il faut également signaler les gongs ou "nugara ", immenses tambours à signaux, en bois, d'une seule pièce comme les pirogues. Chez les Mangbettus ils sont presque toujours munis de deux fortes poignées sculptées, représentant une tête d'antilope, destinées à en faciliter le transport. En expédition, les indigènes emportent des " nugaras " beaucoups plus légers, moins encombrants, ayant exactement la forme du claque, porté au commencement de notre siècle, par les élégants connus sous le nom d'incroyables. L'usage du tabouret en bois sculpté est très répandu chez les Mangbettus, surtout parmi le beau sexe; chaque femme a le sien et l'emporte avec elle partout où elle ra. Le siège de ces petits meubles, légèrement éridé, afin de procurer une assiette plus surre et plus commode, est soutenu par un pied unique, sur l'exécution duquel le sculpteur a concentré toutes les ressources de son art; le tout est ordinairement orné de clous à tête de cuivre, de fabrication européenne. Nous arons eu l'occasion de nous étonner devant une œuvre en bois, réellement remarquable, l'ancien plat de cérémonie du roi Munza. Taillé d'un seul bloc, il se composait d'une auge occupant le centre, flanquée de deux auges latérales plus petites. L'ensemble, qui mesurait environ 2 mètres de long sur 80 centimètres de large, était supporté par un pied ouvragé de $\delta 0$ centimètres de hauteur. Le compartiment du milieu était destiné à recevoir les aliments solides, viandes, poissons, bananes, manioc, etc., composant le repas du sultan; les compartiment extrêmes recevaient les aliments liquides, tels que le miel, l'huile de palme, d'arachides et ainsi de suite. Cette pièce historique de l'ancien mobilier de la couronne de la dynastie Mang- 
bettu, servait de lavabo au clief de poste de Suroango. Lorsque nous avons voulu l'envoyer en Europe, elle arait disparu, emportée sans doute comme une relique sacrée par un des admirateurs du grand Munza, et toutes les recherches faites pour la retrouver furent vaines.

L'industrie de l'ivoire se borne à la confection de trompes de guerre servant aux signaux et à celles des épingles dont le Mangbettu aime orner sa coiffure. Il faut convenir que si les objets fabriqués en cette matière ne présentent pas une grande variété, ils sont en revanche travaillés arec beauconp de gout et arec beaucoup de talent.

Nous voici arrivés à la conclusion logique qu'il y a lieu de tirer de ce qui précède. Nous n'en voyons guère qu'une seule possible :

Par sa grande fertilité et en raison des produits naturels qu'elle offre à l'exploitation, favorisée d'un autre côté par la facilité avec laquelle se trouve la main-d'œuvre, le pays des Mangbettus peut être considéré comme région de grande avenir. Une seule condition est à réaliser pour qu'il en soit ainsi : l'établissement d'une communication facile avec la côte.

Quant aux habitants de la contrée, sans partager complètement l'enthousiasme avec lequel Junker parle de leur supériorité, on ne peut leur refuser un niveau intellectuel légèrement au-dessus de celui de la plupart de leurs congénères. Ils sont certainement doués d'un sens artistique développé comparativement à l'état de leur civilisation; la forme des objets qu'ils produisent est souvent originale et élégante, et, à part les clous en cuivre par lesquels ils enlaidissent leurs tabourets, l'ornementation qu'ils emploient est ordinairement pleine de gout. Mais il faut convenir également des défectuosités et des imperfections assez nombreuses que présente l'exécution considérée dans ses détails; et il ne pourrait 
en être autrement, arec les outils primitifs dont ils disposent et dont ils tirent encore une parti étonnant. Lorsque la civilisation les aura dotés d'un outillage plus parfait, et surtout lorsqu'elle les aura amenés à s'en servir; en un mot lorsque leurs dispositions naturelles auront à leur service les moyens perfectionnés dont nous disposons nous mèmes, il est certain qu'ils marcheront à grands pas dans la voie du progrès et ne tarderont pas à produire des chefs-d'œurre.

Sous les autres rapports, les Mangbettus ne diffèrent pas sensiblement des autres noirs. Ils ont des qualités nombreuses et diverses, parmi lesquelles il s'en trouve un certain nombre de mauvaises; et, pour atteindre notre but final, pour réussir à les élever à notre niveau social, nous derons commencer par encourager leurs bonnes qualités, tout en nous attachant à atténuer progressivement les inconvénients qui résultent de leurs défauts; nous parviendrons ainsi insensiblement à corriger ceus-ci et peut-être même à les faire disparaître en grande partie. 

